\title{
Long Term Clinical Outcomes in Patients with Moderate Aortic Stenosis
}

\author{
Han-Joon Bae, MD, PhD, ${ }^{1,2}$ Jongmin Hwang, MD, PhD, ${ }^{1}$ Seongwook Han, MD, PhD, ${ }^{1}$ \\ Seung-Ho Hur, MD, PhD, ${ }^{1}$ Jin-Wook Chung, MD, PhD,${ }^{3}$ Hyungseop Kim, MD, PhD ${ }^{1}$
}

${ }^{1}$ Division of Cardiology, Department of Internal Medicine, Keimyung University Dongsan Medical Center, Daegu, Republic of Korea; ${ }^{2}$ Division of Cardiology, Department of Internal Medicine, Daegu Catholic University, Medical Center, Daegu, Republic of Korea; ${ }^{3}$ Division of Cardiology, Department of Internal Medicine, Keimyung University Daegu Dongsan Hospital, Daegu, Republic of Korea

\section{ABSTRACT}

Background: While the surgical correction of moderate aortic stenosis (AS) can be deferred with a watchful waiting according to the present guideline, the clinical outcomes for moderate AS with comorbidity have not extensively been studied. We aimed to explore the factors that would contribute to the outcomes of moderate AS with at least five years of follow-up duration.

Methods: Medical records review identified patients with moderate aortic valve (AV) stenosis from January 2008 and December 2012. Echocardiographic data were gathered, and the final 5-year clinical outcomes, defined as the composite of cardiovascular (CV) death, admission for heart failure (HF) aggravation, and AV replacement, were evaluated.

Results: Among 148 patients (mean age, 69.3 years; mean $\mathrm{AV}$ area, $1.24 \mathrm{~cm}^{2}$ ), 79 had adverse outcomes (16 CV deaths, $32 \mathrm{AV}$ replacements, and $31 \mathrm{HF}$ cases), during a mean follow-up of 5.6 years. The event group showed worse dyspnea of NYHA III-IV and a higher frequency of diabetes mellitus (DM). They had a higher frequency of moderate or moderate-to-severe functional mitral regurgitation (MR) and smaller AV area. In the multivariate analysis, DM (HR 2.29, 95\% CI 1.03-5.10), moderate or moderate-to-severe MR (HR 4.84, 95\% CI 1.66-10.07), and NYHA III-IV (HR $3.84,95 \%$ CI 1.72-8.56) independently were associated with adverse outcomes.

Conclusions: The symptomatic patients with moderate AS had higher events than expected, and early intervention should be considered in case of concomitant MR and DM.

\section{INTRODUCTION}

The likelihood of developing aortic stenosis (AS) increases with age and thus, the degenerative changes contribute to

Received March 17, 2020; received in revised form May 1, 2020; accepted May 1,2020 .

Correspondence: Hyungseop Kim, MD, PhD, Division of Cardiology, Department of Internal Medicine, Keimyung University Dongsan Medical Center, 56 Dalsung-ro, 7ung-gu, Daegu 41931 Republic of Korea; +82-53250-7998; fax: +82-53-250-7034 (e-mail: khyungseop@dsmc.or.kr). the common etiology of AS in the elderly population [Passik 1987]. Severe AS requires surgical correction in cases of left ventricular (LV) dysfunction or presence of symptoms, such as dyspnea, angina, or syncope [Nishimura 2017]. Given that perioperative mortality is higher in these patients, the risk and benefit should be considered [Rosenhek 2012]. However, for high surgical risk patients, transcatheter aortic valve implantation (TAVI), together with the surgical advancement of sutureless aortic valve replacement (SU-AVR), has been available, and the indications for these treatments are expanding to patients with moderate risk for surgical correction [Nishimura 2017; Baumgartner 2017].

Nevertheless, the treatment of strategy for moderate AS with complicated comorbidity remains unclear. More importantly, clinical outcomes of moderate AS can be worse than expected, and the risk of a cardiovascular (CV) event during watchful waiting was not exactly left with nothing [Rosenhek 2004]. Considering the advancement of TAVI or SU-AVR, early AS correction appears to be appropriate before the occurrence of adverse events, during a watchful waiting or follow up in moderate AS patients who have LV systolic dysfunction, instead of blindly waiting for reaching to the time of AS correction addressed in the recently released guideline [Baumgartner 2017; van Gils 2017; Spitzer 2016]. Therefore, this study aimed to evaluate the clinical outcomes of patients with moderate AS and to determine the associated factors that could contribute to these outcomes.

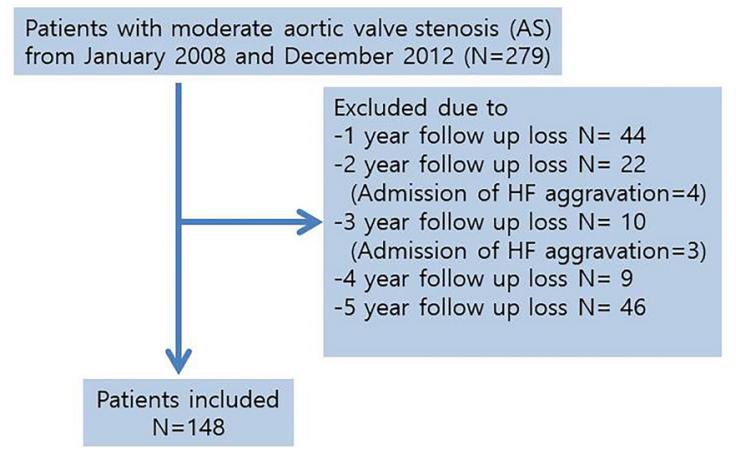

Figure 1. Patients enrollment. 


\section{METHODS}

Study patients: All study participants were identified through a thorough review of the medical records. The eligible patients were referred to the echocardiography laboratory at a single tertiary university medical center for evaluation of valvular heart disease from January 2008 to December 2012. The inclusion criteria of the present study were as follows: 1) age $>18$ years, 2) AS patients with moderate grade, and 3) no or any secondary or functional regurgitation
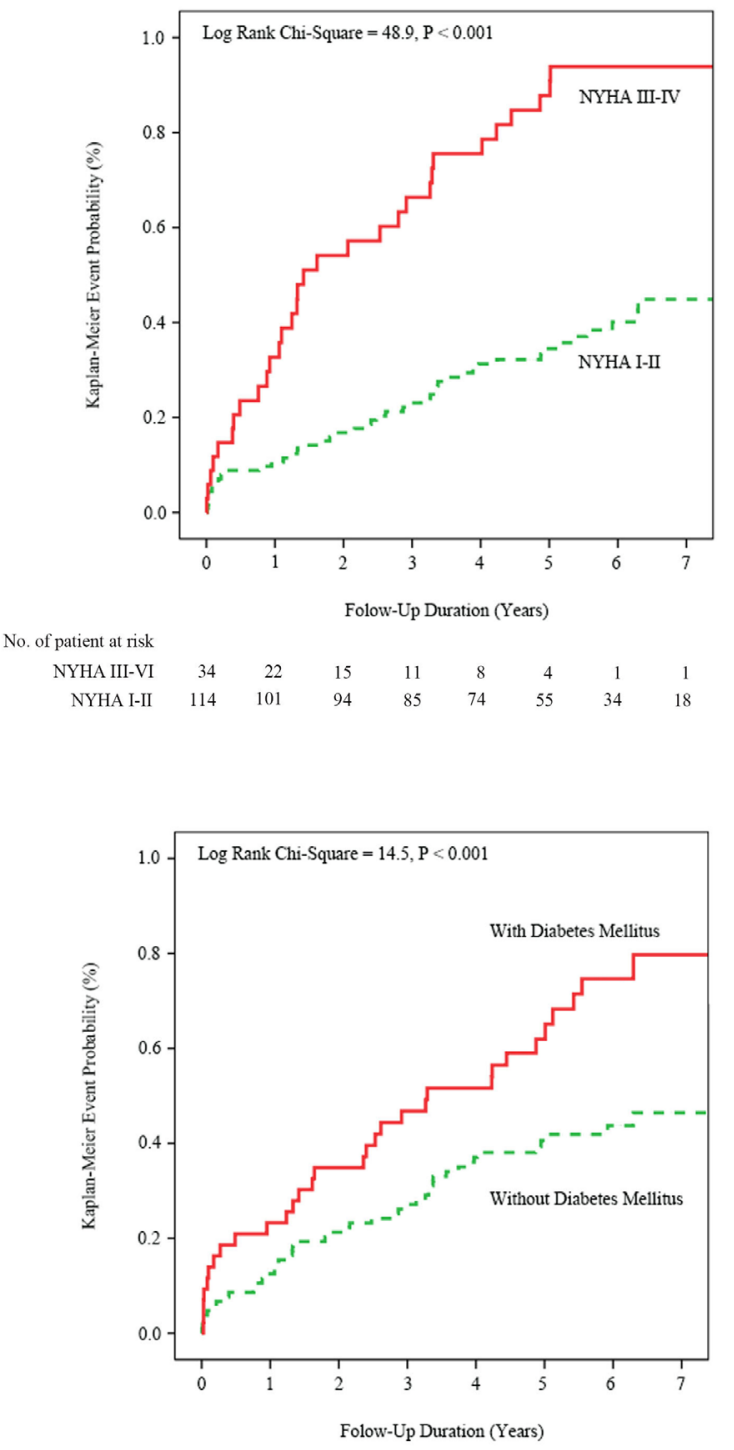

No. of patient at risk

$\begin{array}{rrrrrrrrr}\text { With DM } & 43 & 33 & 28 & 22 & 20 & 12 & 6 & 4 \\ \text { Without DM } & 105 & 90 & 81 & 74 & 62 & 47 & 29 & 15\end{array}$

or stenotic valvular disease (but AV) less than or equal to moderate-to-severe grade. According to the 2017 American Society of Echocardiography clinical recommendations for AV stenosis [Baumgartner 2017], moderate AS was defined if any one of the three criteria was met: peak aortic jet velocity (Vmax) between 3.0 and $4.0 \mathrm{~m} / \mathrm{s}$ on Doppler echocardiography, mean transvalvular pressure gradient between 30 and $40 \mathrm{mmHg}$, and aortic valve area by continuity equation between 1.0 (aortic valve area index more than $0.6 \mathrm{~cm}^{2} / \mathrm{m}^{2}$ ) and $1.5 \mathrm{~cm}^{2}$. Patients with mild or severe AS grade; with primary or intrinsic severe
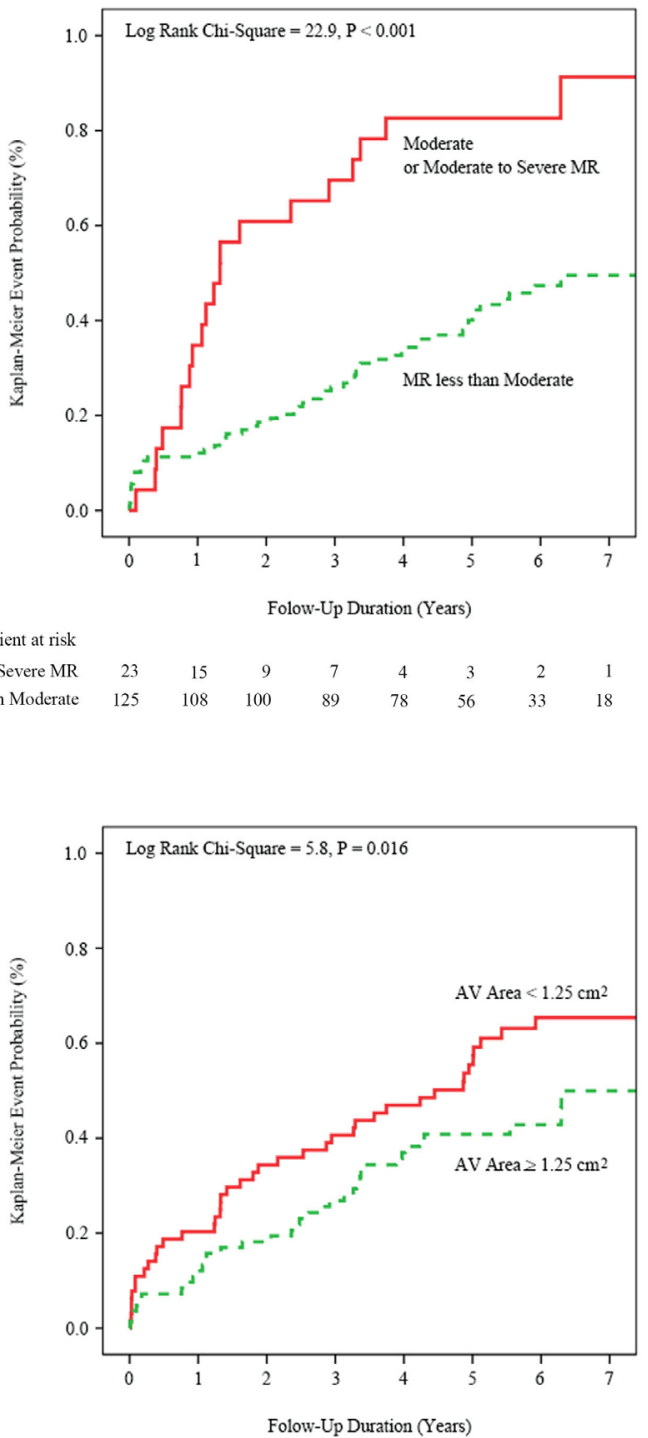

No. of patient at risk

AV Area $<1.25 \mathrm{~cm}^{2} \quad 64 \quad 51 \quad 42 \quad 38 \quad 33 \quad 24 \quad 14 \quad 4$

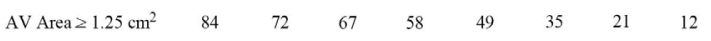

Figure 2. Kaplan-Meier event free curves are shown according to the (A) NYHA functional class, (B) mitral regurgitation of more than the moderate grade, (C) diabetes mellitus, and (D) aortic valve area of $1.25 \mathrm{~cm}^{2}$. 
Table 1. Baseline clinical characteristics of the study population according to the clinical outcomes

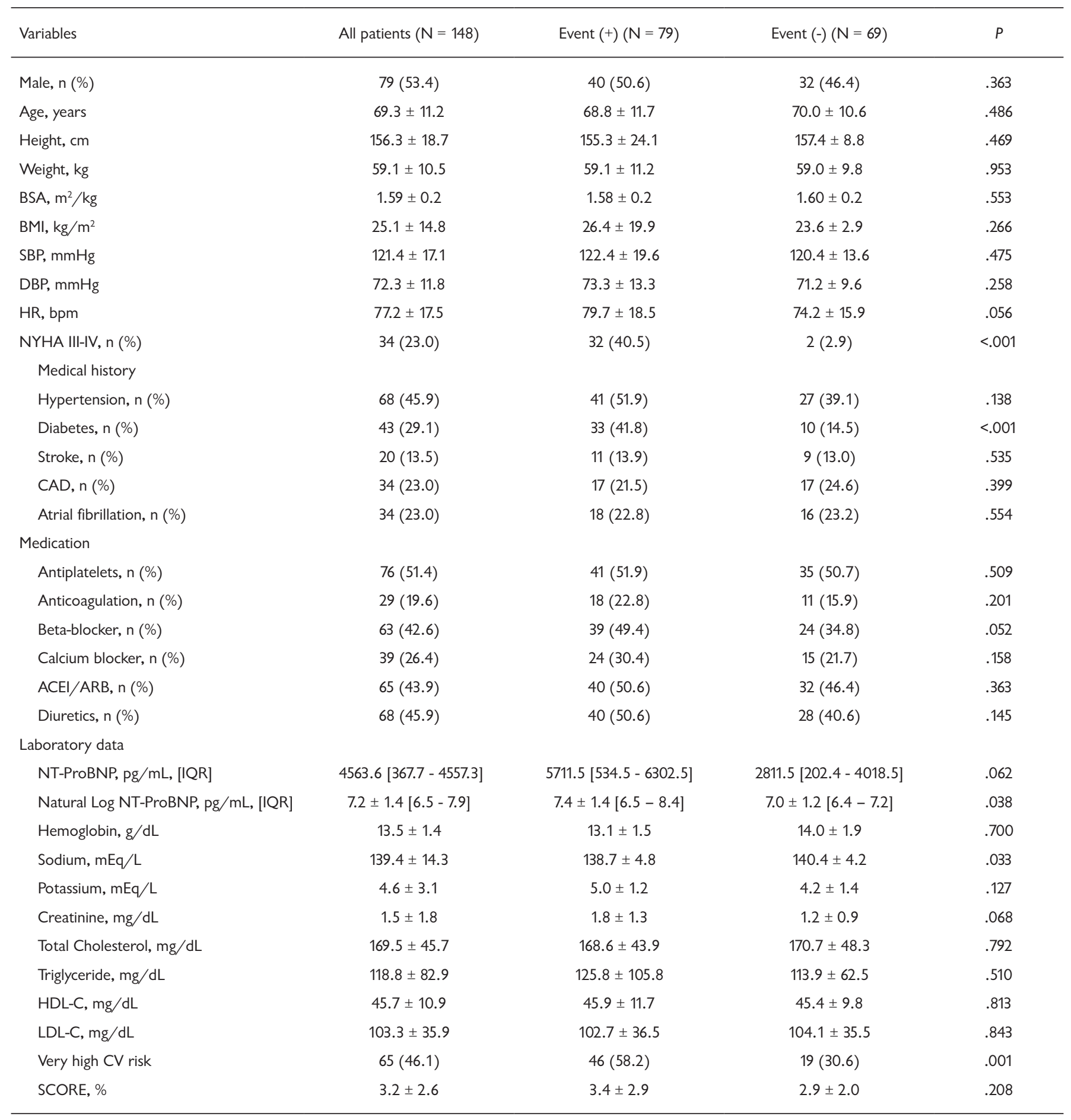

ACEl, Angiotensin-converting enzyme inhibitors; ARB, Angiotensin receptor blocker; BMI, body mass index; BSA, body surface area; CAD, coronary artery diseases (previous angina, previous myocardial infarction, previous coronary artery bypass graft); CV risk, CV risk was classified by risk categories of 2016 ESC/EAS Guidelines for the Management of Dyslipidaemias; DBP, diastolic blood pressure; HDL-C, High density lipoprotein-cholesterol; HR, heart rate; IQL, interquartile range; LDL-C, Low density lipoprotein-cholesterol; NT-ProBNP, N-terminal pro b-type natriuretic peptide; NYHA, New York Heart Association; SCORE, 10-year risk of fatal CVD in population at high CVD risk without high-density lipoprotein-cholesterol (http://www. HeartScore.org); SBP, systolic blood pressure. 


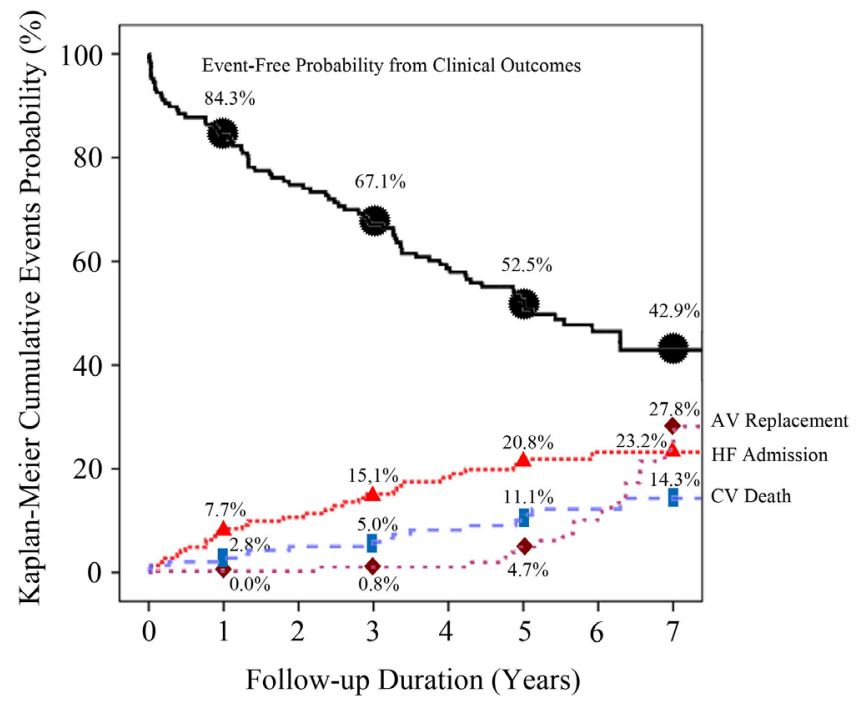

Figure 3. Kaplan-Meier curve of the cumulative probability in moderate aortic stenosis patients is demonstrated, according to the composite of primary end-point for follow-up duration.

valvular disorder other than $\mathrm{AV}$; who underwent surgical correction of any valvular disease; had suffered a dyspnea with New York Heart Association (NYHA) functional class IV; had renal replacement therapy, such as dialysis or transplantation; or had malignancy or active systemic inflammation or infection were excluded from the current analysis. Of 279 patients who were first screened, 131 were excluded because of the incompleteness of minimum follow-up requirements of five years in cases with absent CV clinical outcomes. Finally, 148 patients were included in the analysis. This study protocol conforms to the ethical guidelines of the 1975 Declaration of Helsinki as reflected in a prior approval by the institution's human research committee. The institutional review board approved this research and a written informed consent was waived because of the retrospective nature of this study (Figure 1).

Echocardiography: All patients underwent comprehensive echocardiography, and their data were retrieved from the echocardiography databases at our hospital between January 2008 and December 2012. The echocardiographic parameters were obtained, according to the recommendations of the guidelines [Lang 2015]. LV volumes and ejection fraction (LVEF) were measured using the biplane Simpson's method at apical two-and four-chamber views. Left atrial (LA) volume was calculated using the biplane area-length method's formula, and LA volume index was obtained by dividing LA volume by body surface area. Mitral annular peak systolic and early diastolic tissue Doppler velocities were obtained at the septal annulus on the apical four-chamber view. In relation with $\mathrm{AV}$ severity assessment, $\mathrm{AV}$ peak velocity and mean pressure gradients were measured using the view showing the maximal value. Further, AV area was calculated by continuity equation and indexed to body surface area. The valvular disorders other than $\mathrm{AV}$ were presented as a severity of moderate or moderate-to-severe grade.
Clinical outcomes: The primary endpoint of this study was a composite of CV death, AV replacement, and hospitalization for worsening heart failure after the index echocardiography. The worsening HF was identified, according to the Framingham Heart Study criteria, and ascertained by a requirement of hospitalization and using intravenous diuretics to relieve dyspnea and pulmonary edema demonstrated by chest X-ray. CV-related death was confirmed by a review of patient's medical record or phone calls with next of kin. The first event was considered when any event from composite occurred during the follow-up duration. Follow-up data were evaluated for primary outcomes by reviewing medical records or through telephone interviews. The 5-year follow-up completeness and CV events were $100 \%$ and $53.4 \%$, respectively, which were obtained by the index devised by Clark et al [Clark 2002]. The study patients were followed for a mean of $5.6 \pm 2.4$ years after the index date with respect to the first event.

Statistical analysis: The descriptive data were showed as a number (percent) for categorical variables, and as the mean \pm standard deviation for continuous variables of normal distribution, or median (interquartile range [IQR]) in cases of non-normal distribution. The chi-square test or Student's t-test was performed for comparison between two groups, as appropriate. Prior to entry into Cox models, raw BNP values were natural $\log$ transformed. The significant variables in the univariate Cox analysis were entered into the multivariate model, and the associated factors with primary endpoints were identified. Cumulative event probability or event-free survival analysis was performed using the Kaplan-Meier test. All of the statistical analyses were performed with the Statistical Package for Social Science version 23.0. (SPSS, IBM Corporation, Armonk, New York, USA). A $P$-value $<.05$ was considered statistically significant.

\section{RESULTS}

Baseline characteristics of the whole study population: The baseline characteristics of the patients are listed in Table 1. The mean age was $69.3 \pm 11.2$ years, and $53.4 \%$ were male. Among the enrolled patients, $45.9 \%$ had hypertension, $29 \%$ had diabetes mellitus (DM), 23\% had NYHA functional class III dyspnea, and $23 \%$ had atrial fibrillation. The median value of NTproBNP was $4563.6 \mathrm{pg} / \mathrm{mL}$ (IQR 367.7-4557.3). In relation to primary endpoint, among a total of 148 patients, the clinical outcomes occurred in $79(53.4 \%)$ patients over a mean followup period of 5.6 years, with $16 \mathrm{CV}$ deaths, $32 \mathrm{AV}$ replacements, and $31 \mathrm{HF}$ cases. In the primary endpoint, there were no significant differences, in terms of age, the medical histories of hypertension, stroke, atrial fibrillation, and coronary artery disease, or their medications between the event and no event groups. However, the event group showed higher frequencies of NYHA III-IV and DM, and lower level of serum sodium concentration, compared with the no event group.

Echocardiographic data: Patients with events had significantly larger LV end-systolic volume, and there also was a trend toward an increase in end-diastolic volume (Table 2). They had higher pulmonary artery systolic pressure and E/e' ratio. The 
prevalence of moderate or moderate-to-severe $\mathrm{MR}$ was higher among patient with events, although LA size was not significantly different between the two groups. Regarding AV study, AV-area/area index was smaller in the event group, whereas the other $\mathrm{AV}$ indices, such as velocity, time-velocity integral, or mean pressure gradient, were not significantly different between the two groups.
Association between concomitant risk factors and clinical events: During a mean follow-up period of 5.6 years, univariate parameters for clinical outcomes were DM, NYHA III$\mathrm{IV}, \mathrm{AV}$ area $<1.25 \mathrm{~cm}^{2}$, and moderate or moderate-to-severe MR (Table 3). The Kaplan-Meier curve suggested that the event rate in patients with a moderate or moderate-to-severe

Table 2. Echocardiographic data of the study population according to the clinical outcomes

\begin{tabular}{|c|c|c|c|c|}
\hline Variables & All patients $(\mathrm{N}=148)$ & Event $(+)(\mathrm{N}=79)$ & Event $(-)(N=69)$ & $P$ \\
\hline LVEDD, cm & $5.04 \pm 0.75$ & $5.09 \pm 0.88$ & $4.98 \pm 0.57$ & .371 \\
\hline $\mathrm{LAD}, \mathrm{cm}$ & $4.60 \pm 1.01$ & $4.59 \pm 1.02$ & $4.60 \pm 1.01$ & .953 \\
\hline LVEF, \% & $59.5 \pm 14.5$ & $58.6 \pm 14.9$ & $60.2 \pm 14.3$ & .505 \\
\hline LVESV-4C, mL & $54.0 \pm 54.4$ & $66.2 \pm 67.6$ & $39.1 \pm 25.8$ & .036 \\
\hline LVEDV-2C, mL & $104.4 \pm 60.2$ & $114.2 \pm 71.7$ & $92.5 \pm 40.3$ & .132 \\
\hline LVESV-2C, mL & $53.5 \pm 51.9$ & $66.4 \pm 61.8$ & $37.8 \pm 30.6$ & .020 \\
\hline IVST, cm & $1.00 \pm 0.02$ & $1.00 \pm 0.20$ & $1.01 \pm 0.17$ & .541 \\
\hline PWT, cm & $0.99 \pm 0.02$ & $0.99 \pm 0.20$ & $1.00 \pm 0.16$ & .761 \\
\hline \multicolumn{5}{|l|}{ Mitral inflow study } \\
\hline E flow, $\mathrm{m} / \mathrm{s}$ & $0.95 \pm 0.44$ & $1.10 \pm 0.53$ & $0.85 \pm 0.34$ & .139 \\
\hline DT, ms & $278.5 \pm 138.1$ & $279.8 \pm 149.0$ & $277.1 \pm 126.0$ & .909 \\
\hline IVRT, ms & $102.9 \pm 23.3$ & $98.2 \pm 19.2$ & $108.3 \pm 26.4$ & .009 \\
\hline$E / e^{\prime}$ & $20.9 \pm 13.2$ & $23.5 \pm 16.4$ & $18.3 \pm 8.5$ & .041 \\
\hline $\mathrm{s}^{\prime}, \mathrm{cm} / \mathrm{s}$ & $6.16 \pm 1.70$ & $5.91 \pm 1.88$ & $6.43 \pm 1.46$ & .076 \\
\hline $\mathrm{e}^{\prime}, \mathrm{cm} / \mathrm{s}$ & $5.46 \pm 2.12$ & $5.46 \pm 2.47$ & $5.46 \pm 1.71$ & .099 \\
\hline Moderate or moderate-to-severe AR, n (\%) & $35(23.6)$ & $14(17.7)$ & $21(30.4)$ & .052 \\
\hline Moderate or moderate-to-severe MR, n (\%) & $25(16.9)$ & $20(25.3)$ & $5(7.2)$ & $<.001$ \\
\hline Mean PG, $\mathrm{mmHg}$ & $24.7 \pm 5.5$ & $24.7 \pm 5.5$ & $24.7 \pm 5.6$ & .989 \\
\hline AVA, $\mathrm{cm}^{2}$ & $1.24 \pm 0.15$ & $1.20 \pm 0.13$ & $1.29 \pm 0.15$ & $<.001$ \\
\hline AVA index, $\mathrm{cm}^{2} / \mathrm{m}^{2}$ & $0.80 \pm 0.13$ & $0.77 \pm 0.13$ & $0.83 \pm 0.13$ & .009 \\
\hline
\end{tabular}

2C, 2-chamber; 4C, 4-chamber; AR, aortic regurgitation; AV, aortic valve; AVA, aortic valve area; DT, deceleration time; IVRT, isovolumic relaxation time; IVST, interventricular septal thickness; LA, left atrium; LAD, left atrium dimension; LAVI, left atrium volume index; LV, left ventricle; LVEDD, left ventricle end diastolic dimension; LVEDV, left ventricular end diastolic volume; LVESD, left ventricle end systolic dimension; LVESV, left ventricular end systolic volume; LVEF, left ventricle ejection fraction; LVOT, left ventricle outflow tract; MR, mitral regurgitation; MS, mitral stenosis; PASP, pulmonary artery systolic pressure; PG, pressure gradient; PWT, left ventricular posterior wall thickness; TR, tricuspid regurgitation; TVI, time velocity integral. 
Table 3. Univariate and multivariate Cox hazard regression analyses for the clinical outcomes

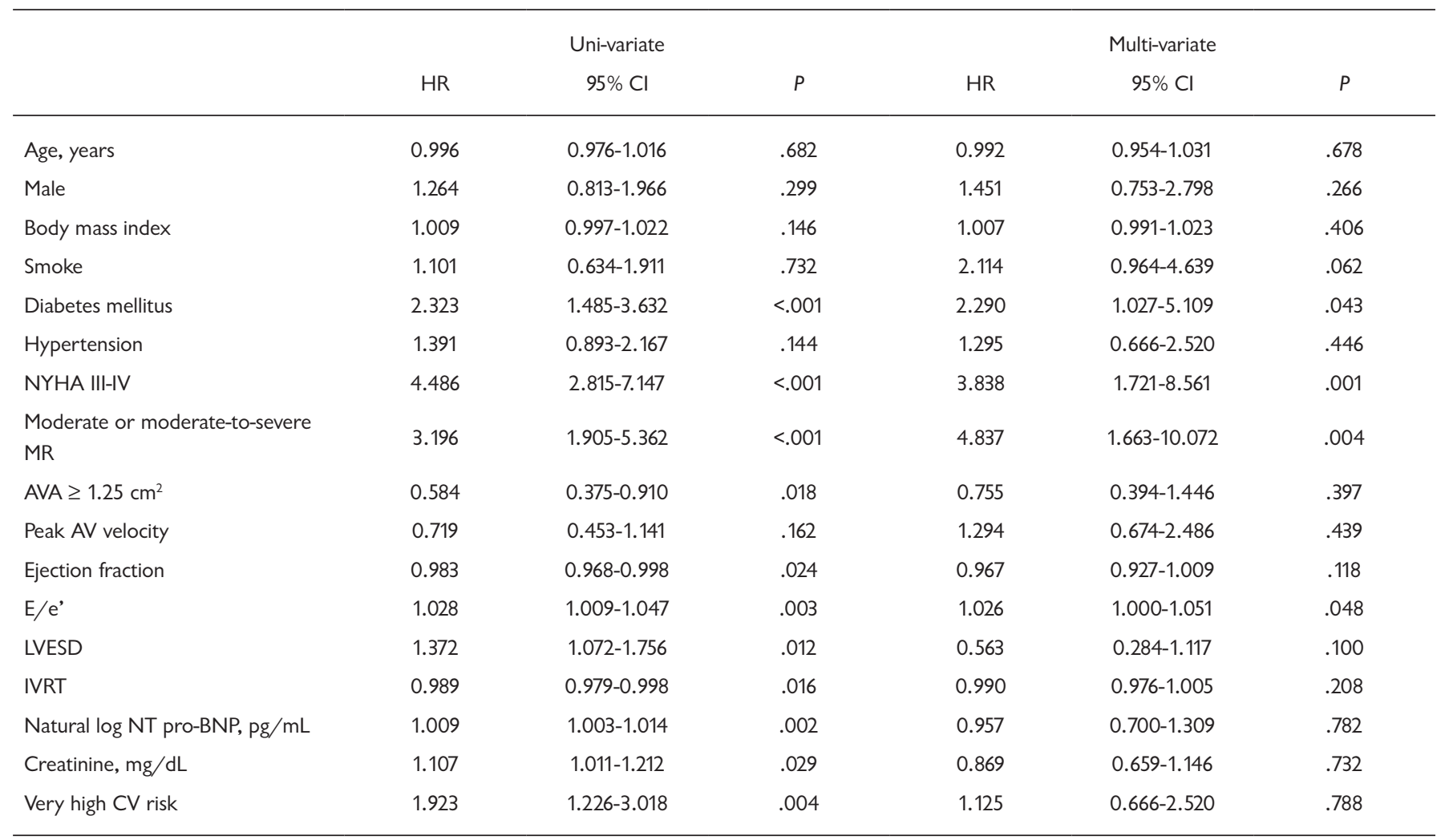

AV, aortic valve; AVA, aortic valve area; CV risk, CV risk was classified by risk categories of 2016 ESC/EAS Guidelines for the Management of Dyslipidaemias; NT-ProBNP, N-terminal pro b-type natriuretic peptide; NYHA, New York Heart Association Functional Classification; MR, mitral regurgitation.

MR, or NYHA III-IV is significantly higher after 6-month follow up, compared with those who have DM or AV area < $1.25 \mathrm{~cm}^{2}$. (Figure 2A to 2D) In a multivariate analysis, DM (HR, 2.29 [95\% CI, 1.03-5.10]), NYHA III-IV (HR, 3.84 [95\% CI, 1.72-8.56]), and moderate or moderate-to-severe MR (HR, 4.84 [95\% CI, 1.66-10.07]) were significantly associated with an increased risk of 5-year events (Table 3). According to the cumulative freedom probability from the primary endpoint, AV replacement was performed predominantly after five years of follow-up duration, although the increase in the number of HF admission and $\mathrm{CV}$ death steadily was observed after the index echocardiography (Figure 3).

\section{DISCUSSION}

This study reported the adverse outcome of moderate AS, during a follow-up period of 5.6 years. The main findings of the present study are as follows: 1 ) The primary endpoint of $\mathrm{CV}$ deaths, hospitalization for $\mathrm{HF}$, or $\mathrm{AV}$ replacement occurred in $53.4 \%$ of moderate AS patients; and 2) The degree equal to or more than moderate MR, history of DM, and NYHA III-IV dyspnea independently were associated with adverse events.
Impact of mitral regurgitation on cardiac dysfunction in moderate aortic stenosis: Adverse outcomes are overtly occurring even in moderate AS, but there are no clear guidelines for their therapeutic strategies. The presence of moderate or moderate-to-severe MR in this population results in cardiac dysfunction and LV volume overload, leading to $\mathrm{LV}$ fibrosis on top of pressure overloading of AS per se [Ahmed 2010; El Sabbagh 2018]. This is because that LV end-systolic volume on echocardiographic two-/four-chamber views was greater in the event group. Along with an enlarged LV end-systolic volume, LA volume and E/e' ratio also are basically considered as indicators of diastolic dysfunction [Nagueh 2016]. In proportion to the diastolic dysfunction, higher pulmonary artery systolic pressure (PASP) could be explained.

However, in the current study, most of the patients with moderate AS already had enlarged LA or indexed LA volume, making it difficult to differentiate between patients with CV events and those without. Furthermore, moderate AS patients failed to show the difference of a conventional LVEF between the two groups, although LVEF has been regarded as an important surgical indicator for severe AS in asymptomatic patients. As such, it seems likely that the worse diastolic dysfunction aggravated by significant MR hemodynamics would be a more important contributing factor rather than LVEF. 
[SUPPLEMENT TBL 1] Concomitant disease, reason for surgery, and method of aortic valve replacement

\begin{tabular}{lcccc}
\hline AVR $(\mathrm{N}=32)$ & Severe AS $(\mathrm{N}=18)$ & Moderate AS $(\mathrm{N}=14)$ & & \\
Method & $\operatorname{TAVR}(\mathrm{N}=2)$ & $\operatorname{SAVR}(\mathrm{N}=16)$ & $\operatorname{TAVR}(\mathrm{N}=0)$ & $\operatorname{SAVR}(\mathrm{N}=14)$ \\
Concomitant disease & - & CABG 3, MVR 1 & - & Aneurysm 1, CABG 2, Severe AR 2, Severe MS 2, Severe MR 2, \\
Unknown 5
\end{tabular}

Clinical presentation and moderate aortic stenosis: According to the present study, most of the AV replacement seemed to be deferred until five years. This partly could be due to a watchful waiting or the uncertain belief or consensus in relation to moderate, not severe AS. The most important thing is that the clinical events occurred before AV replacement. One of the major symptoms associated with AS is dyspnea on exertion, which could reflect the severe AS grade. Frequently, dyspnea or HF symptoms do not occur until the AS is very severe. However, even in moderate AS, dyspnea still can be present when there are concomitant valvular disorders with increased LV end-diastolic pressure caused by significant MR. Accordingly, in patients with dyspnea not explained just by moderate AS, unfavorable prognosis can be expected, and earlier surgical correction is helpful, as has been demonstrated in patients with the multi-valvular disorders.

Dyspnea can be a clinical marker that indicates the timing for surgical correction, and LV dysfunction has been regarded as a poor risk factor [van Gils 2017; Yechoor]. The comorbidity, such as DM, NYHA III-IV, or ischemic heart disease, can affect the natural course of AS. In the present study, an elevated PASP, increased LA volume, presence of MR of more than the moderate grade, and an elevated E/e' ratio can contribute to the development of dyspnea. Indeed, dyspnea could be aggravated by other multifactorial factors, in addition to AS.

Clinical outcomes of moderate aortic stenosis: In the natural course of AS, the event rate of moderate AS even without symptoms seemed to be significantly higher in the previously reported data [Yechoor]. The cohort of asymptomatic moderate AS shows an event rate of nearly $35 \%-40 \%$ from the general population [Bahler 2018]. Concomitant MR, particularly that with more than the moderate grade, can be a challenging problem, in terms of determining the need for surgical correction. According to the present guideline, there is no clear consensus of the therapeutic strategy for multi-valvular disorders, in terms of early surgical correction or watchful waiting until reaching the severe AS grade.

Among the AV parameters, only AV area or indexed area was a significant risk factor in univariate analysis. The Doppler parameters associated with hemodynamics would underestimate the severity of AV stenosis because the forward stroke volume could be reduced by concomitant MR, leading to a low mean pressure gradient across AV. Despite the importance of calculated AV area, accurately calculating AV area in patients with an elliptical-shaped LV outflow tract may be difficult. These may explain the lack of significance of the $\mathrm{AV}$ area in multivariate analysis.

It is worth noting that the event group reveals much more unfavorable LV hemodynamics caused by multi-valvular disorders, including an increased DM, PASP and elevated E/e' ratio, which can add microvascular coronary dysfunction, stressful pressure to $\mathrm{LV}$ function in addition to coexistence of valvular heart disease [Benfari 2018; Banovic 2016]. Therefore, an underestimated severity of AS can be expected only when using a mean pressure gradient or AV peak velocity, in case an appropriate AV area cannot be calculated. Given the interrelation between MR and AS hemodynamics and low mortality of early correction with advanced technique [Nishimura 2017], early AV correction would be an appropriate therapeutic option before the occurrence of irreversible LV or LA dysfunction caused by concomitant comorbidities.

Particularly, these patients with comorbid LV dysfunction could get the benefit of a 5-year event-free period from an early durable AV correction, when considering the Kaplan-Meier cumulative event rate in the present study. The high event rate in these patients should be considered for further research and may justify the early intervention in this population. In real-world practice, it is challenging to determine timing for valvular correction in multiple and mixed valvular heart disease, particularly low-pressure and low-gradient AS which can frequently be observed in case of concomitant significant MR. Accordingly, a timely adequate correction of AS can enhance or improve the LV remodeling with the decrease in MR observed [Catapano 2016; Unger 2008]. Those therapeutic strategies are highly required in patients with balanced severity of valve lesion, short interval between follow-up visits, and frequent serial evaluation by a multidisciplinary heart valve team [Sehovic 2015].

Limitations: There are several important limitations that should be addressed in this study. First, this is a single center retrospective study; thus, a well-organized prospective study with more data should be required in the future. Second, a fastidious quantitative study about valvular disorder, including mitral or aortic regurgitation, was not performed. However, in this study, the presence of moderate or moderate-to-severe MR, not minimal or mild grade, could be enough for a physician to determine the significant grade affecting or causing $\mathrm{LV}$ dysfunction or hemodynamics. Third, the number of other comorbidities is not enough and thus, it is difficult to make a clear decision about early intervention, when there is a comorbidity. Last, this study did not include patients with bicuspid AV. Therefore, our findings cannot be applied to bicuspid AV disease.

\section{CONCLUSION}

Although moderate AS does not meet the current surgical indication, the presence of worsening dyspnea or MR greater 
than moderate grade can affect the prognosis of AS. Therefore, in moderate AS patients, the evaluation of risk factors worsening the natural course of AS would be useful to guide an appropriate strategy for patients who may benefit from early valvular correction.

\section{REFERENCES}

Ahmed MI, Gladden JD, Litovsky SH, Lloyd SG, Gupta H, Inusah S, et al. 2010. Increased oxidative stress and cardiomyocyte myofibrillar degeneration in patients with chronic isolated mitral regurgitation and ejection fraction > 60\%. J Am Coll Cardiol 55:671-679.

Bahler RC, Hawi R, Rovner A, Finkelhor RS, Thomas CL, Dawson NV. 2018. Predicting Outcomes in Patients With Asymptomatic Moderate to Severe Aortic Stenosis. Am J Cardiol 122:851-858.

Banovic M, Brkovic V, Nedeljkovic I, Popovic D, Djordjevic-Dikic A, Ristic A, et al. 2016. Diabetes mellitus and coronary microvascular function in asymptomatic patients with severe aortic stenosis and nonobstructed coronary arteries. Diab Vasc Dis Res 13:220-227.

Baumgartner H, Falk V, Bax JJ, De Bonis M, Hamm C, Holm PJ, Iung B, et al. 2017. 2017 ESC/EACTS Guidelines for the management of valvular heart disease. Eur Heart J 38:2739-2791.

Baumgartner H, Hung J, Bermejo J, Chambers JB, Edvardsen T, Goldstein S, 2017. Recommendations on the Echocardiographic Assessment of Aortic Valve Stenosis: A Focused Update from the European Association of Cardiovascular Imaging and the American Society of Echocardiography. J Am Soc Echocardiogr 30:372-392.

Benfari G, Nistri S, Faggiano P, Clavel MA, Maffeis C, Enriquez-Sarano M, et al. 2018. Mitral Effective Regurgitant Orifice Area Predicts Pulmonary Artery Pressure Level in Patients with Aortic Valve Stenosis. J Am Soc Echocardiogr 31:570-577.

Catapano AL, Graham I, De Backer G, Wiklund O, Chapman MJ, Drexel H, et al. 2016. 2016 ESC/EAS Guidelines for the Management of Dyslipidaemias. Eur Heart J 37:2999-3058.

Clark TG, Altman DG, De Stavola BL. 2002. Quantification of the completeness of follow-up. Lancet 359:1309-10.

El Sabbagh A, Reddy YNV, Nishimura RA. 2018. Mitral Valve Regurgitation in the Contemporary Era: Insights Into Diagnosis, Management, and Future Directions. JACC Cardiovasc Imaging 11:628-643.

Lang RM, Badano LP, Mor-Avi V, Afilalo J, Armstrong A, Ernande L, et al. 2015. Recommendations for cardiac chamber quantification by echocardiography in adults: an update from the American Society of Echocardiography and the European Association of Cardiovascular Imaging. Eur Heart J Cardiovasc Imaging 16:233-270.

Nagueh SF, Smiseth OA, Appleton CP, Byrd BF 3rd, Dokainish H, Edvardsen T, et al. 2016. Recommendations for the Evaluation of Left Ventricular Diastolic Function by Echocardiography: An Update from the American Society of Echocardiography and the European Association of Cardiovascular Imaging. Eur Heart J Cardiovasc Imaging 17:1321-1360.

Nishimura RA, Otto CM, Bonow RO, Carabello BA, Erwin JP 3rd, Fleisher LA, et al. 2017. 2017 AHA/ACC Focused Update of the 2014 AHA/ACC Guideline for the Management of Patients With Valvular Heart Disease: A Report of the American College of Cardiology/American Heart Association Task Force on Clinical Practice Guidelines. J Am Coll Cardiol 70:252-289.

Passik CS, Ackerann DM, Pluth JR, Edwards WD. 1987. Temporal changes in the causes of aortic stenosis: a surgical pathologic study of 646 cases. Mayo Clin Proc 62:119-123.

Rosenhek R, Iung B, Tornos P, Antunes MJ, Prendergast BD, Otto CM, et al. 2012. ESC Working Group on Valvular Heart Disease Position Paper: assessing the risk of interventions in patients with valvular heart disease. Eur Heart J 33:822-828.

Rosenhek R, Klaar U, Schemper M, Scholten C, Heger M, Gabriel H, et al. 2004. Mild and moderate aortic stenosis. Natural history and risk stratification by echocardiography. Eur Heart J 25:199-205.

Sehovic S, Talic A, Kacila M, Tahirovic E. 2015. The Influence of Aortic Valve Replacement on Functional Moderate - To-Severe Mitral Regurgitation in Patients with Aortic Valve Stenosis. Acta Inform Med 23:147-150.

Spitzer E, Van Mieghem NM, Pibarot P, Hahn RT, Kodali S, Maurer MS, et al. 2016. Rationale and design of the Transcatheter Aortic Valve Replacement to UNload the Left ventricle in patients with ADvanced heart failure (TAVR UNLOAD) trial. Am Heart J 182:80-88.

Unger P, Plein D, Van Camp G, Cosyns B, Pasquet A, Henrard V, et al. 2008. Effects of Valve Replacement for Aortic Stenosis on Mitral Regurgitation. Am J Cardiol 102:1378-1382.

van Gils L, Clavel MA, Vollema EM, Hahn RT, Spitzer E, Delgado V, et al. 2017. Prognostic Implications of Moderate Aortic Stenosis in Patients With Left Ventricular Systolic Dysfunction. J Am Coll Cardiol 69:2383-2392.

Yechoor P, Blaustein AS, Bakaeen FG, Cornwell LD, Coselli JS, LeMaire SA, et al. The natural history of moderate aortic stenosis in a veteran population. J Thorac Cardiovasc Surg 145:1550-1553. 